\title{
Practice and awareness of physicians regarding casual-clinic blood pressure measurement in Japan
}

\author{
Mitsuru Kobayashi ${ }^{1,7}$, Taku Obara ${ }^{1,2,7}$, Takayoshi Ohkubo ${ }^{1,3}$, Hidefumi Fukunaga ${ }^{3}$, Michihiro Satoh ${ }^{1}$, \\ Hirohito Metoki ${ }^{1,4}$, Kei Asayama ${ }^{3}$, Ryusuke Inoue ${ }^{5}$, Masahiro Kikuya ${ }^{1}$, Nariyasu Mano ${ }^{2}$, \\ Masaaki Miyakawa ${ }^{6}$ and Yutaka Imai ${ }^{1}$
}

The optimal procedure for casual-clinic blood pressure (CBP) measurement is outlined in the 2004 Japanese guidelines. We investigated the status of physicians' practices and their awareness of CBP measurement immediately and 4 years after the publication of the guidelines using a questionnaire regarding CBP. This survey was conducted among physicians who attended educational seminars on hypertension in 2004-2005 and in 2007-2008; the questionnaire was distributed, completed and collected just before the start of the seminars. Of the 1966 respondents to the 2004-2005 survey and the 2995 respondents to the 2007-2008 survey, the proportion of physicians who answered that CBP was more important than self-measured BP at home (home BP) was less than $10 \%$ in both surveys. The proportion of physicians who used a mercury sphygmomanometer (68.1-75.5\%) was higher than those who used an automatic and electronic sphygmomanometer (20.7-29.0\%) in both surveys. However, the use of an automatic and electronic sphygmomanometer slightly increased from $20.7 \%$ in 2004-2005 to $29.0 \%$ in 2007-2008. Physicians who were younger or working in a hospital were less likely to measure CBP using the conditions of the guidelines. Approximately $50 \%$ of physicians correctly recognized the reference values of hypertension based on CBP measurement (systolic/diastolic, $140 / 90 \mathrm{~mm} \mathrm{Hg}$ ) in both surveys. The status of physicians' practice and awareness of CBP measurement varied by physicians' age, specialty and work place and those situations did not change over 4 years, underlying the importance of clarifying factors inherent in these situations.

Hypertension Research (2010) 33, 960-964; doi:10.1038/hr.2010.89; published online 10 June 2010

Keywords: casual-clinic blood pressure; guidelines; physicians

\section{INTRODUCTION}

Blood pressure (BP) is a major vital sign used to evaluate the physical condition of each patient. Measurement of casual-clinic BP (CBP) in clinical settings has been used as the gold standard for hypertension evaluation. Under the Japanese guidelines for the management of hypertension, the optimum conditions for CBP measurement include the use of the auscultation method, with a mercury or aneroid sphygmomanometer for the measurement of CBP, or the use of an automatic sphygmomanometer that has been calibrated. ${ }^{1,2}$ Previous reports have shown that $\mathrm{CBP}$, as measured in strict accordance with the procedures set forth in the guidelines, had a clinical value at least comparable with BP measured in out-of-clinical settings, ${ }^{3}$ although BP measured in out-of-clinical settings has several advantages, including the avoidance of both observer bias and the white-coat effect. ${ }^{4,5}$ Therefore, in clinical trials and epidemiological surveys, CBP has often been measured in accordance with the procedures outlined in the guidelines. However, in actual clinical settings, CBP may rarely be measured in accordance with the guidelines and the accuracy of CBP measurement may be ignored. In addition, physicians' compliance with the guidelines' recommendations has not been evaluated.

The objective of this study is to investigate and compare the status of physicians' practice, awareness of CBP measurement based on the 2004 Japanese guidelines and compliance with the guidelines immediately and 4 years after publication of the guidelines.

\section{METHODS}

A questionnaire survey regarding self-measured BP at home (home BP; HBP)/ CBP was conducted among physicians who attended educational seminars on hypertension in 32 Japanese prefectures from September 2004 to June 2005 and in 40 prefectures from November 2007 to July 2008. This questionnaire was distributed and collected just before the start of the seminars. The questionnaire

${ }^{1}$ Department of Clinical Pharmacology and Therapeutics, Tohoku University Graduate School of Pharmaceutical Sciences and Medicine, Sendai, Japan; ${ }^{2}$ Department of Pharmacy, Tohoku University Hospital, Sendai, Japan; ${ }^{3}$ Department of Planning for Drug Development and Clinical Evaluation, Tohoku University Graduate School of Pharmaceutical Sciences, Sendai, Japan; ${ }^{4}$ Department of Medical Genetics, Tohoku University Graduate School of Medicine, Sendai, Japan; ${ }^{5}$ Medical Informatics, Tohoku University Graduate School of Medicine, Sendai, Japan and ${ }^{6}$ Miyakawa Clinic, Yokohama, Japan

${ }^{7}$ These authors contributed equally to this work.

Correspondence: Dr T Ohkubo, Department of Planning for Drug Development and Clinical Evaluation, Tohoku University Graduate School of Pharmaceutical Sciences, 2-1 Seiryo-cho, Aoba-ku, Sendai 980-8575, Japan.

E-mail: tohkubo@mail.tains.tohoku.ac.jp

Received 9 December 2009; revised 19 April 2010; accepted 28 April 2010; published online 10 June 2010 
included questions relating to the device used for CBP measurement, the condition and environment of CBP measurement, the number of CBP measurements taken during each visit, and the physician's awareness of the reference values for CBP measurement. Of the 2190 physicians who attended the educational seminar in 2004-2005, 1966 (89.8\%) responded; for the seminar held in 2007$2008,2995(87.7 \%)$ of the 3416 physicians in attendance responded.

\section{RESULTS}

The characteristics of the physicians attending the seminars were comparable between surveys (Table 1). Older physicians were more likely to be male and be working in a clinic in both surveys. The proportion of physicians who answered that CBP was more important than HBP was less than $10 \%$ (7.8\% in $2004-2005,4.4 \%$ in 2007-2008). Physicians who regarded CBP as more important than HBP were more likely to be older internists who often worked in a clinic. The proportion of physicians who asked their patients to measure BP in a waiting room by themselves using an automated device (apart from CBP measurement performed by a nurse or a physician) was $31.3 \%$ in $2004-2005$ and $32.6 \%$ in $2007-2008$. The proportion of physicians who introduced self-measurement of CBP by patients was inversely related to physicians' age.

The proportion of physicians who used a mercury sphygmomanometer was approximately $70 \%$ in both surveys and slightly decreased in 2007-2008 (Figure 1). On the other hand, the proportion of physicians who used an automatic and electronic sphygmomanometer for CBP measurement was approximately $25 \%$ in both surveys but slightly increased in 2007-2008 (Figure 1).

Approximately $43 \%$ of physicians measured CBP only once at each visit. Slightly fewer (approximately 35\%) physicians measured CBP twice each visit (Figure 2). Approximately $90 \%$ of physicians measured CBP in the seated position, and another $30 \%$ measured BP after more than 5 min of rest. Approximately $15 \%$ of physicians measured CBP under the restrictions of no caffeine consumption and no smoking, whereas $28 \%$ of physicians measured CBP in a room with controlled temperature. An additional $45 \%$ of physicians measured CBP without conversation during the BP measurement (Table 2). These proportions were comparable between surveys (Table 2).

The proportion of physicians who correctly recognized the reference values for hypertension based on CBP was approximately $60 \%$ for

\section{Table 1 Characteristics of physicians}

\begin{tabular}{lcc}
\hline & $\begin{array}{c}2004-2005 \\
(\mathrm{n}=1966)\end{array}$ & $\begin{array}{c}2007-2008 \\
(\mathrm{n}=2995)\end{array}$ \\
\hline $\begin{array}{l}\text { Gender } \\
\text { Male, (\%) }\end{array}$ & 90.8 & 90.2 \\
Age & & \\
$<40$ years, (\%) & 13.9 & 11.0 \\
$40-49$ years, (\%) & 28.2 & 27.2 \\
$50-59$ years, (\%) & 27.4 & 33.8 \\
$60-69$ years, (\%) & 14.2 & 13.0 \\
$\geqq 70$ years, (\%) & 16.3 & \\
Specialty & & 82.6 \\
Internal medicine, (\%) & & \\
& 83.2 & 32.4 \\
Work place & & 65.2 \\
Hospital (public, private), (\%) & & \\
Clinic, (\%) & 39.8 & \\
\hline
\end{tabular}

both systolic and diastolic BP, respectively, and approximately $50 \%$ for systolic/diastolic BP, in both surveys (Table 3).

Differences in response to the questionnaires varied according to physicians' gender, age, specialty and work place in each survey, as shown in Table 4, which is comprised of the combined data from both surveys. Physicians were more likely to use mercury sphygmomanometer for CBP measurement if they worked in a clinic, regarded CBP as more important than HBP or were internists. Physicians who were older or worked in a clinic were more likely to measure CBP under conditions set forth in the guidelines. Physicians who were younger, internists or worked in a hospital were more likely to know the reference values for CBP hypertension. Compared to younger physicians, older physicians were more likely to answer the value $<140 \mathrm{~mm} \mathrm{Hg}$ as the reference value for systolic hypertension (physicians' age $<40$ years, $40-49,50-59,60-69$ and $\geqslant 70$ years; $12,17,22$, 22 and $32 \% ; P<0.0001)$ and the value $<90 \mathrm{~mm} \mathrm{Hg}$ as the reference value for diastolic hypertension $(19,23,29,31$ and $47 \%$; $P<0.0001)$.

\section{DISCUSSION}

We found that physicians' compliance with the guidelines regarding the optimum procedure for CBP measurement was poor and did not differ significantly between the two surveys. We also found that compliance with the guidelines in Japan varied according to

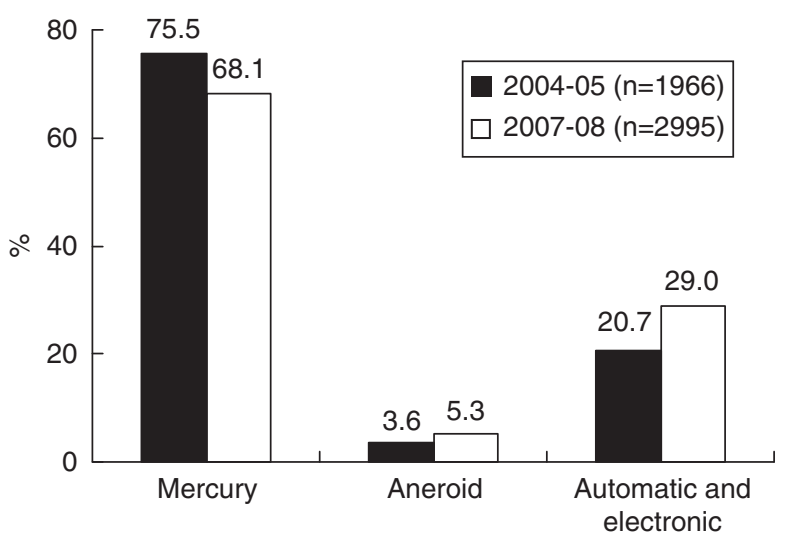

Figure 1 Responses to the question 'Which sphygmomanometers do you use to measure patients' CBP? CBP, casual-clinic blood pressure.

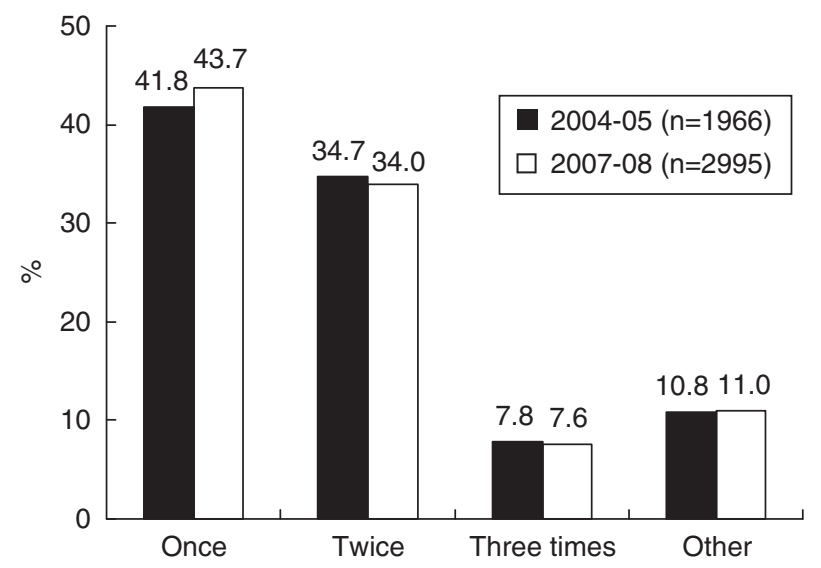

Figure 2 Responses to the question 'How many times do you measure CBP in each visit?' CBP, casual-clinic blood pressure. 
Table 2 Practice of CBP measurement

\begin{tabular}{|c|c|c|}
\hline & $\begin{array}{c}2004-2005 \\
(n=1966)\end{array}$ & $\begin{array}{c}2007-2008 \\
(n=2995)\end{array}$ \\
\hline \multicolumn{3}{|l|}{ (a) Body position (multiple choice) } \\
\hline Sitting position, (\%) & 87.7 & 89.2 \\
\hline Recumbent position, (\%) & 15.4 & 14.7 \\
\hline Standing position, (\%) & 0.9 & 0.6 \\
\hline \multicolumn{3}{|c|}{ (b) Five minutes or more of rest before measurement } \\
\hline Followed, (\%) & 32.0 & 30.5 \\
\hline Not followed, (\%) & 39.4 & 40.3 \\
\hline Noncommittal, (\%) & 24.2 & 25.6 \\
\hline No answer, (\%) & 4.4 & 3.6 \\
\hline \multicolumn{3}{|c|}{ (c) Smoking and taking caffeine before measurement } \\
\hline Restricted, (\%) & 15.2 & 14.2 \\
\hline Unrestricted, (\%) & 48.1 & 50.1 \\
\hline Depending on the situation, (\%) & 11.6 & 12.4 \\
\hline Not considered, (\%) & 20.2 & 18.1 \\
\hline No answer, (\%) & 4.9 & 5.2 \\
\hline \multicolumn{3}{|l|}{ (d) Room temperature } \\
\hline Considered, (\%) & 27.4 & 29.2 \\
\hline Unconsidered, (\%) & 26.3 & 26.8 \\
\hline Noncommittal, (\%) & 7.6 & 8.8 \\
\hline Common temperature, (\%) & 32.3 & 29.1 \\
\hline Has not been considered, (\%) & 6.1 & 6.1 \\
\hline Other, (\%) & 0.3 & 0.0 \\
\hline \multicolumn{3}{|l|}{ (e) Conversation during measurement } \\
\hline Yes, (\%) & 35.7 & 35.2 \\
\hline No, (\%) & 45.7 & 46.1 \\
\hline Noncommittal, (\%) & 13.9 & 14.9 \\
\hline No answer, (\%) & 4.7 & 3.8 \\
\hline
\end{tabular}

Abbreviation: CBP, casual-clinic blood pressure.

Table 3 Awareness of the reference values of hypertension for CBP

\begin{tabular}{lcc}
\hline & $\begin{array}{c}2004-2005 \\
(\mathrm{n}=1966)\end{array}$ & $\begin{array}{c}2007-2008 \\
(\mathrm{n}=2995)\end{array}$ \\
\hline (a) Systolic blood pressure & & 20.9 \\
$<140 \mathrm{~mm} \mathrm{Hg},(\%)$ & 20.9 & 58.8 \\
$140 \mathrm{~mm} \mathrm{Hg},(\%)$ & 63.0 & 8.0 \\
$>140 \mathrm{~mm} \mathrm{Hg},(\%)$ & 6.8 & 12.3 \\
No answer, (\%) & 9.3 & \\
& & 28.3 \\
(b) Diastolic blood pressure & & 55.9 \\
$<90 \mathrm{~mm} \mathrm{Hg},(\%)$ & 29.7 & 3.3 \\
$90 \mathrm{~mm} \mathrm{Hg},(\%)$ & 58.0 & 12.6 \\
$>90 \mathrm{~mm} \mathrm{Hg},(\%)$ & 2.9 & \\
No answer, (\%) & 9.5 & 49.9 \\
\hline (c) Systolic/diastolic blood pressure & & \\
$140 / 90 \mathrm{~mm}$ Hg, (\%) & 52.1 &
\end{tabular}

Abbreviation: CBP, casual-clinic blood pressure.

physicians' age, specialty and work place. In addition, we reviewed the CBP measuring condition in foreign countries and found that detailed surveys regarding the CBP measuring condition in routine clinical settings have not been reported in foreign countries. Some foreign studies, however, have compared CBP values measured in routine clinical practice to those measured in accordance with the guidelines. All reported that CBP values measured in routine clinical practice were higher compared to those measured in accordance with the guidelines. ${ }^{6-8}$ Therefore, poor adherence to guidelines regarding the optimum procedure for CBP measurement may be associated with higher CBP values, not only in Japan, but also in foreign countries.

A mercury sphygmomanometer was the most frequently used BP device in Japan. However, the use of the mercury sphygmomanometer has slightly decreased in Japan over time and the use of the automatic and electronic sphygmomanometers is gaining popularity. These tendencies may be explained by the fact that mercury is recognized as an environmental pollutant and accurate automatic electronic sphygmomanometers have been developed over the past decade. ${ }^{9}$

The Japanese guidelines recommend that CBP be measured at least twice and diagnosis of hypertension should be based on the average of two stable values of CBP obtained at multiple visits. ${ }^{1,2}$ In this survey, about $40 \%$ of physicians measured CBP only once in daily practice. According to our analysis from Japan Home versus Office blood pressure Measurement Evaluation (J-HOME) study that conducted in treated hypertensive patients in Japan, ${ }^{10}$ the false-negative rate of the first BP measure was negligible (0.7\%); however, the false-positive rate was nearly one in four (23\%).' Therefore, some patients may be inadequately evaluated if their BP is assessed based solely on a single CBP measurement.

The proportion of physicians who measured CBP after more than 5 min of rest and without conversation during CBP measurement were only about 30 and $45 \%$, respectively. These findings may reflect the busy practices of Japanese practitioners, especially in the hospital setting. The BP measurement conditions regarding no conversation during CBP measurement and measurement of CBP after more than 5 min of rest also might be affected by the number of patients cared for per day. Ignoring these two conditions may lead to misdiagnosis and inappropriate control of hypertensive patients and possibly cause white-coat hypertension. However, since most physicians answered that HBP is equally or more important than CBP and recommended that their patients measure HBP, these physicians might manage their patients based on HBP values.

As it has been reported that smoking can affect BP levels, ${ }^{11,12}$ the consideration of smoking status is important in evaluating patients' BP. However, the proportion of physicians who restricted smoking before CBP measurement was small. These results may reflect the fact that physicians no longer need to restrict patients' smoking before CBP measurement, because smoking has recently been banned in almost all medical settings in Japan. However, as smoking is one of the factors affecting hypertension in out-of-medical settings such as masked hypertension, ${ }^{13}$ smoking status should be considered in the evaluation of patients' total cardiovascular risk. ${ }^{1,2}$ The proportion of physicians who took the temperature of the room into consideration when measuring CBP was only approximately $30 \%$. As room temperature is managed in almost all hospitals or clinics, the answer may vary depending on whether the responder is an administrator of a hospital or a clinic. In fact, in this survey, the proportion of physicians who took room temperature into consideration was higher among those who worked in a clinic compared with those who worked in a hospital.

Half of the physicians correctly recognized the reference values for CBP hypertension. Until a decade ago, the reference values for CBP hypertension were $160 / 95 \mathrm{~mm} \mathrm{Hg}$ for systolic/diastolic BP; ${ }^{14}$ thus, it is to be expected that older physicians would tend to answer with 
Table 4 Responses to questions by physicians according to gender, age, work place, specialty and preference of BP measurement

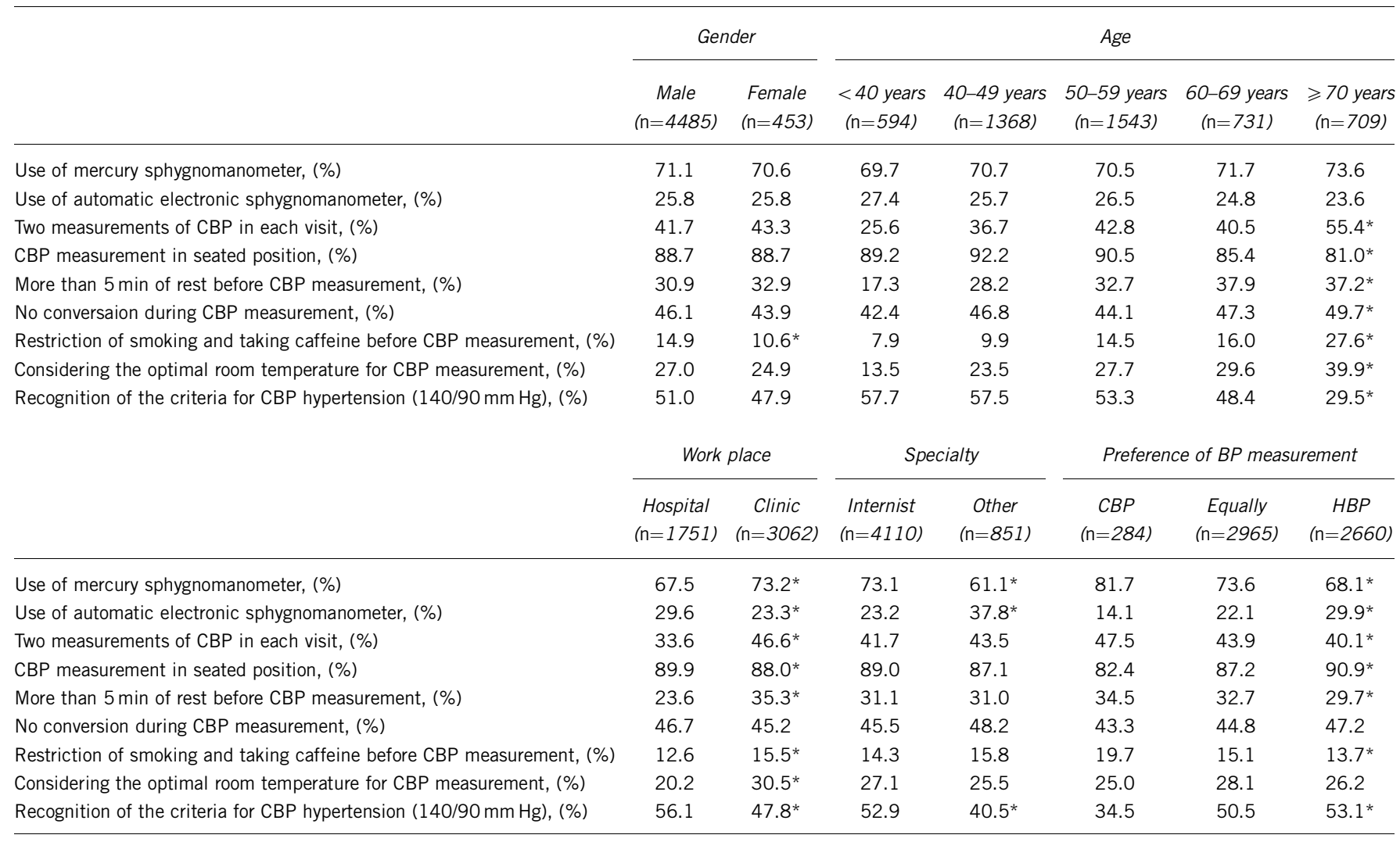

Abbreviations: BP, blood pressure; CBP, casual-clinic blood pressure; HBP, home blood pressure;

${ }^{*} P<0.05$ among each subgroup.

a higher value than $140 / 90 \mathrm{~mm} \mathrm{Hg}$ for systolic/diastolic BP as the reference values of hypertension. ${ }^{15}$ However, surprisingly, the proportion of physicians who answered with values lower than 140/ $90 \mathrm{~mm} \mathrm{Hg}$ for systolic/diastolic BP was higher among older physicians than younger physicians. These results might be partly explained by the target BP levels for young to middle-aged patients and patients with renal diseases, diabetes or ischemic heart diseases in the Japanese guidelines. ${ }^{1,2}$

Physicians who did not measure CBP under the procedures set forth in the guidelines were more likely to be young and often worked in a hospital. Physicians who recommended to their patients that they measure HBP and asked them to measure their BP by themselves in the waiting room using an automatic device were also more likely to be young and often worked in a hospital. As younger physicians and physicians working in hospitals are frequently busy, these physicians might introduce HBP measurements and self-measurements of BP in the office. A previous study showed that the correlation coefficient between awake ambulatory BP (systolic/diastolic) and BP self-measured by patients using an automated device $(r=0.62 / 0.72)$ was significantly higher than the coefficient measured between awake ambulatory BP and BP measured by physicians using a manual sphygmomanometer $(r=0.32 / 0.48){ }^{6}$ Therefore, BP self-measured in a waiting room by patients using an automated device may be more accurate compared with BP measured by physicians in a clinic. However, the target BP levels based on HBP measurements and the optimal procedure for self-measurement of BP in a waiting room have not yet been established. Therefore, at this time, it is essential that physicians measure CBP using the procedures suggested in the guide- lines to correctly evaluate their patients' BP and manage their hypertension.

This study had several limitations. As candidates in this study were physicians attending educational seminars on hypertension, they may have been biased because they had a greater interest in the diagnosis and treatment of hypertension regardless of the actual amount of their knowledge concerning hypertension, when compared with average Japanese general physicians. Although the number of patients cared for per day or week might affect the degree of physicians' compliance with optimal procedure of CBP measurement set forth in the guidelines, we did not collect the information on the number of patients cared for per day or week in the present study. The changes in physicians' practice and awareness of CBP measurement over 4 years were not precisely evaluated, because participation in the surveys was done anonymously and thus we did not collect information that would identify physicians who attended both surveys.

We found that the status of physicians' practice and awareness of CBP measurement, in accordance with the guidelines varied according to physicians' age, specialty and work place, and these situations have not changed significantly over 4 years. Further study is needed to identify the reason why physicians who are young or working in a hospital do not measure CBP in accordance with the optimal procedure set forth in the guidelines. At the same time, development of proper hypertension management based on out-of-office BP measurements is needed.

\section{CONFLICT OF INTEREST}

The authors declare no conflict of interest. 


\section{ACKNOWLEDGEMENTS}

This work was supported by directors, councilors and members of the Japanese Society of Hypertension: Kikuo Arakawa, Mikio Arita, Shuji Arima, Katsuyuki Ando, Masao Ishii, Toshihiko Ishimitsu, Sadayoshi Ito, Tsutomu Imaizumi, Hirotsugu Ueshima, Yoshio Uehara, Satoshi Umemura, Nobuyuki Ura, Hidenori Urata, Mitsuru Oishi, Yusuke Ohya, Toshio Ogihara, Tomohiro Osanai, Shigehiro Katayama, Tomohiro Katsuya, Yuhei Kawano, Kenjirou Kikuchi, Kazuo Kitamura, Kenjiro Kimura, Toshio Kushiro, Shunichi Kojima, Ikuo Saito, Atsuhisa Sato, Takao Saruta, Kazuaki Shimamoto, Tatsuo Shimosawa, Hiromichi Suzuki, Kenji Takazawa, Hakuo Takahashi, Kazuhisa Takeuchi, Akira Takeshita, Yasuko Tanabe, Takuya Tsuchihashi, Kimio Tomita, Mitsuhide Naruse, Naoyuki Hasebe, Jitsuo Higaki, Yukio Hirata, Toshiro Fujita, Hideo Matsuura, Hiroaki Matsuoka, Masayasu Matsumoto, Junichi Minami, Minoru Yamakado, Tsutomu Yamasaki, Takeshi Yamashita, Kenichi Yoshida and Hiromi Rakugi, in the order of the Japanese syllabary. This work was supported by a Grant from the National Cardiovascular Research Foundation (H19-Kou8) and a Grant-in-Aid for Japan Society for the Promotion of Science (JSPS) fellows (18.54042 and 20.7477).

1 Japanese Society of Hypertension. Japanese Society of Hypertension guidelines for the management of hypertension (JSH 2004). Hypertens Res 2006; 29(Suppl): S1-S105.

2 Ogihara T, Kikuchi K, Matsuoka H, Fujita T, Higaki J, Horiuchi M, Imai Y, Imaizumi T, Ito S, Iwao H, Kario K, Kawano Y, Kim-Mitsuyama S, Kimura G, Matsubara H, Matsuura H, Naruse M, Saito I, Shimada K, Shimamoto K, Suzuki H, Takishita S, Tanahashi N, Tsuchihashi T, Uchiyama M, Ueda S, Ueshima H, Umemura S, Ishimitsu T, Rakugi H, Japanese Society of Hypertension Committee. The Japanese Society of Hypertension Guidelines for the Management of Hypertension (JSH 2009). Hypertens Res 2009; 32: 3-107.

3 Fagard RH, Staessen JA, Thijs L. Prediction of cardiac structure and function by repeated clinic and ambulatory blood pressure. Hypertension 1997; 29: 22-29.
4 Imai Y, Ohkubo T, Hozawa A, Tsuji I, Matsubara M, Araki T, Chonan K, Kikuya M, Satoh H, Hisamichi S, Nagai K. Usefulness of home blood pressure measurements in assessing the effect of treatment in a single-blind placebo-controlled open trial. $J$ Hypertens 2001; 19: 179-185.

5 Ohkubo T, Asayama K, Kikuya M, Metoki H, Hoshi H, Hashimoto J, Totsune K, Satoh H, Imai Y, Ohasama Study. How many times should blood pressure be measured at home for better prediction of stroke risk? Ten-year follow-up results from the Ohasama study. $J$ Hypertens 2004; 22: 1099-1104.

6 Myers MG, Oh P, Reeves RA, Joyner CD. Prevalence of white coat effect in treated hypertensive patients in the community. Am J Hypertens 1995; 8: 591-597.

7 Brown MA, Buddle ML, Martin A. Is resistant hypertension really resistant? Am J Hypertens 2001; 14: 1263-1269.

8 Graves JW, Nash C, Burger K, Bailey K, Sheps SG. Clinical decision making in hypertension using an automated (BpTRU) measurement device. J Hum Hypertens 2003; 17: 823-827.

9 Shirasaki O, Terada H, Niwano K, Nakanishi T, Kanai M, Miyawaki Y, Souma T, Tanaka T, Kusunoki T. The Japan Home-health Apparatus Industrial Association: investigation of home-use electronic sphygmomanometers. Blood Press Monit 2001; 6: 303-307.

10 Ohkubo T, Obara T, Funahashi J, Kikuya M, Asayama K, Metoki H, Oikawa T, Takahashi H, Hashimoto J, Totsune K, Imai Y, J-HOME Study Group. Control of blood pressure as measured at home and office, and comparison with physicians' assessment of control among treated hypertensive patients in Japan: First Report of the Japan Home versus Office Blood Pressure Measurement Evaluation (J-HOME) study. Hypertens Res 2004; 27: 755-763.

11 Groppeli A, Giorgi DMA, Omboni S, Parati G, Mancia G. Persistent blood pressure increase induced by heavy smoking. J Hypertens 1992; 10: 495-499.

12 Niedermaier ON, Smith ML, Beightol LA, Zukowska-Grojec Z, Goldstein DS, Eckberg DL. Influence of cigarette smoking on human autonomic function. Circulation 1993; 88: 562-571.

13 Kawano Y, Horio T, Matayoshi T, Kamide K. Masked hypertension: subtypes and target organ damage. Clin Exp Hypertens 2008; 30: 289-296.

14 Ministry of Health and Welfare, Japan/Japan Medical Association. Handbook of hypertension. Nippon Ishikai Zasshi 199; 10: 1-89 (in Japanese).

15 Ikeda N, Hasegawa T, Hasegawa T, Saito I, Saruta T. Awareness of the Japanese Society of Hypertension Guidelines for the Management of Hypertension (JSH 2000) and compliance to its recommendations: surveys in 2000 and 2004. J Hum Hypertens 2006; 20: 263-266.

\section{APPENDIX}

\section{Questionnaire}

Appendix

Questionnaire

1. Gender

2. Age

1. Male $\quad$ 2. Female

1. 20-29 years 2. 30-39 years $3.40-49$ years $4.50-59$ years

5. 60-69 years 6. 70-79 years 7.80 years or more

3. Specialty

$\begin{array}{ll}\text { 1. Internal medicine } & \text { 2. Surgery } \quad \text { 3. Other }\end{array}$

4. Work place

1. Hospital (public, private) 2. Clinic 3. Other

5. Which $\mathrm{BP}$ is important when you judge or treat patients with hypertension? $\begin{array}{lll}\text { 1. CBP } & \text { 2. HBP } & \text { 3. Equally important }\end{array}$

6. Do you ask patients to measure $\mathrm{BP}$ in a waiting room by themselves using an automated device?

$\begin{array}{ll}\text { 1. Yes } & \text { 2. No }\end{array}$

7. Which sphygmomanometers do you use to measure patients' CBP? (Multiple-choice)

$\begin{array}{ll}\text { 1. Mercury sphygmomanometer } & \text { 2. Aneroid sphygmomanometer }\end{array}$

3. Automatic electronic sphygmomanometer

8. How many times do you measure CBP in each visit?

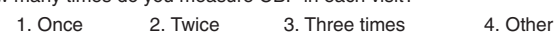

9. Please select your practice of CBP measurement for each condition listed below.

a. Body position (Multiple-choice)

$\begin{array}{ll}\text { 1. Sitting position } & \text { 2. Recumbent position }\end{array}$

3. Standing position

b. Five minutes or more of rest before measurement

$$
\begin{array}{llll}
\text { 1. Followed } & \text { 2. Not followed } & \text { 3. Noncommittal }
\end{array}
$$

c. Smoking and taking caffeine before measurement

$\begin{array}{ll}\text { 1. Restricted } & \text { 2. Unrestricted }\end{array}$

3. Depending on the situation 4. Not considered

d. Room temperature

$$
\begin{array}{lll}
\text { 1. Considered } & \text { 2. Not considered } & \text { 3. Noncommittal }
\end{array}
$$

4. Common temperature 5. Has not been considered

e. Conversation during measurement
1. Yes
2. No
3. Noncommittal

10. Please indicate the reference values of hypertension for $\mathrm{CBP}$ recommended by the JSH 2004 and JSH 2000 guidelines. Systolic ( ) $\mathrm{mmHg}$ Diastolic ( $) \mathrm{mmHg}$ HBP, home blood pressure; CBP, casual-clinic blood pressure; JSH, Japanese Society of Hypertension. 